\title{
Non-Cayley-Tree Model for Quasiparticle Decay in a Quantum Dot
}

\author{
X. Leyronas, J. Tworzydło, and C. W. J. Beenakker \\ Instituut-Lorentz, Universiteit Leiden, P.O. Box 9506, 2300 RA Leiden, The Netherlands
}

(Received 2 December 1998)

\begin{abstract}
The decay of a quasiparticle in a confined geometry, resulting from electron-electron interactions, has been mapped onto the single-electron problem of diffusion on a Cayley tree discussed by Altshuler et al. [Phys. Rev. Lett. 78, 2803 (1997)]. We study an alternative model that captures the strong correlations between the self-energies of different excitations with the same number of quasiparticles. The model has a recursion relation for the single-particle density of states that is markedly different from that of the Cayley tree. It remains tractable enough such that sufficiently large systems can be studied to observe a breakdown of the golden rule of perturbation theory with decreasing excitation energy. [S0031-9007(99)09395-3]
\end{abstract}

PACS numbers: 72.15.Lh, 72.15.Rn, 73.23.-b

The lifetime of a quasiparticle in a quantum dot has been the subject of recent experimental [1] and theoretical works [2-9]. Much of the theoretical interest was fueled by the striking prediction of Altshuler, Gefen, Kamenev, and Levitov [3] of a critical excitation energy below which the lifetime becomes essentially infinite. This prediction was based on a mapping between the decay process of a quasiparticle and the phenomenon of Anderson localization on a Cayley tree [10,11]. An infinite lifetime corresponds to the absence of diffusion on the lattice in Fock space consisting of $n$-particle eigenstates $\Psi_{n}$ of the Hamiltonian without interactions. Subsequent theoretical work by Mirlin and Fyodorov [5] and by Jacquod and Shepelyansky [6] indicated that the localization transition is smooth rather than abrupt, extending over a range of excitation energies from $\Delta g^{1 / 2}$ to $\Delta g^{2 / 3}$ (with $\Delta$ the single-particle level spacing and $g$ the conductance in units of $e^{2} / h$ ). The thermodynamic limit $g \gg 1$ is essential for the appearance of the transition.

Numerical diagonalizations of a microscopic Hamiltonian [8] and of the two-body random interaction model [9] were too far from the thermodynamic limit to observe the localization transition. The need for a numerical test of the theory is pressing because of a fundamental difference between the decay process in Fock space and the diffusion process on a Cayley tree. The mapping between the two problems maps different $\Psi_{n}$ 's with the same $n$ onto different sites at the same level of the tree. While in the Cayley tree diffusion from each of these sites is independent; in the Fock space the decay of different $\Psi_{n}$ 's is strongly correlated.

In this paper we consider the model Hamiltonian proposed by Georgeot and Shepelyansky [12], which permits one to study these strong correlations in systems that are bigger than in Refs. [8,9]. We find a smooth transition, signaled by a breakdown of the golden rule of perturbation theory. This is the first observation of the breakdown in a numerical simulation. An analytical approximation to our numerical diagonalizations highlights the origin of the correlations between the $\Psi_{n}$ 's.
The Hamiltonian for spinless fermions is $H=H_{0}+$ $H_{1}$, with

$$
H_{0}=\sum_{j} \varepsilon_{j} c_{j}^{\dagger} c_{j}, \quad H_{1}=\sum_{i<j, k<l} V_{i j, k l} c_{l}^{\dagger} c_{k}^{\dagger} c_{i} c_{j} .
$$

The noninteracting part $H_{0}$ contains the single-particle levels $\varepsilon_{j}$ in a disordered quantum dot. We count the levels from the Fermi level, meaning that the ground state of $H_{0}$ has occupied levels for $j<0$ and empty levels for $j \geq 0$. We assume that an energy level $\varepsilon_{j}$ is uniformly distributed in the interval $\left[\left(j-\frac{1}{2}\right) \Delta,\left(j+\frac{1}{2}\right) \Delta\right]$. This yields a linear level repulsion, consistent with time-reversal symmetry. The basis of $H_{0}$ consists of states that have $m$ electron excitations (occupied levels with $j \geq 0$ ) and $n$ hole excitations (empty levels with $j<0$ ). The two-body interaction $H_{1}$ couples them to states that differ by at most two electron-hole pairs.

We assume that $V_{i j, i j}=0$. (These diagonal terms can be incorporated into $H_{0}$ in a mean-field approximation.) For the off-diagonal matrix elements we adopt the layer model of Ref. [12], which is based on the following observation. The interaction strength $V$ is related to $\Delta$ and $g$ by $[2,3,13] V=\Delta / g$. Since $V \ll \Delta$ for $g \gg 1$, only eigenstates of $H_{0}$ within an energy layer of width $\Delta$ are strongly coupled by the interaction. The layer model exploits this in a clever way by setting $V_{i j, k l}=0$ unless $i, j, k, l$ are four distinct indices with $i+j=k+l$. The nonzero $V_{i j, k l}$ are chosen to be independent real random variables, subject to the restriction $V_{i j, k l}=V_{k l, i j}$ imposed by the Hermiticity of the Hamiltonian. We also set $V_{j i, k l}=-V_{i j, k l}=V_{i j, l k}$. The distribution of each matrix element is taken to be a Gaussian with zero mean and variance $V^{2}$.

One advantage of the layer model is that the ground state $|\mathrm{FS}\rangle$ of $H_{0}$ (the Fermi sea) remains an eigenstate of $H_{0}+H_{1}$. We assume that it remains the ground state. A second advantage is that the effective dimension of the Hilbert space is greatly reduced. The number of states into which an electron excitation $c_{j}^{\dagger}|\mathrm{FS}\rangle$ of energy $\varepsilon_{j}$ decays is 
equal to the number $\mathcal{P}(j) \approx(4 j \sqrt{3})^{-1} \exp (\pi \sqrt{2 j / 3})$ of partitions of $j$ [14], independent of the number $N$ of electrons in the quantum dot (as long as $N>j$ ). This grows much more slowly with $j$ than in the conventional twobody random interaction model $[15,16]$, used in previous work $[6,9,17]$. While the layer model renders the problem tractable, it preserves the strong correlations mentioned in the introduction, as we will discuss shortly.

The decay of the quasiparticle state $c_{j}^{\dagger}|\mathrm{FS}\rangle$ is described by the Green function

$$
\begin{aligned}
G_{j}(E) & =\left\langle\mathrm{FS}\left|c_{j}\left(E+E_{\mathrm{FS}}-H\right)^{-1} c_{j}^{\dagger}\right| \mathrm{FS}\right\rangle \\
& =\left[E-\varepsilon_{j}-\Sigma_{j}(E)\right]^{-1},
\end{aligned}
$$

where $E_{\mathrm{FS}}$ is the energy of the Fermi sea: $H|\mathrm{FS}\rangle=$ $H_{0}|\mathrm{FS}\rangle=E_{\mathrm{FS}}|\mathrm{FS}\rangle$. The second equality in Eq. (2) defines the self-energy $\Sigma_{j}(E)$. The quantity of physical interest (measured by means of a tunneling probe in Ref. [1]) is the single-particle density of states $\rho_{j}(E)=\sum_{\alpha} \delta(E+$ $\left.E_{\mathrm{FS}}-E_{\alpha}\right)\left|\left\langle\alpha\left|c_{j}^{\dagger}\right| \mathrm{FS}\right\rangle\right|^{2}$, where the sum over $\alpha$ runs over all eigenstates $|\alpha\rangle$ of $H$, with eigenvalues $E_{\alpha}$. It is related to the imaginary part of the Green function by

$$
\rho_{j}(E)=-\frac{1}{\pi} \lim _{\eta \rightarrow 0} \operatorname{Im} G_{j}(E+i \eta) .
$$

The ensemble average $\bar{\rho}_{j}(E)$ is not sensitive to the delocalization transition. For that reason, we will also study the inverse participation ratio $P_{j}(E)=\sum_{\alpha} \delta\left(E+E_{\mathrm{FS}}-\right.$ $\left.E_{\alpha}\right)\left|\left\langle\alpha\left|c_{j}^{\dagger}\right| \mathrm{FS}\right\rangle\right|^{4}$, related to the Green function by

$$
P_{j}(E)=\frac{1}{\pi} \lim _{\eta \rightarrow 0} \eta\left|G_{j}(E+i \eta)\right|^{2} .
$$

The dimensionless ensemble-averaged quantity

$$
P_{j}=\bar{P}_{j}\left(\varepsilon_{j}\right) / \bar{\rho}_{j}\left(\varepsilon_{j}\right)
$$

increases from 0 to 1 on going from extended to localized states.

We have computed the Green function numerically using an iterative Lanczos method. The largest system we could study in this way has $j=30$, corresponding to a basis of $\mathcal{P}(30)=5604$ states. Before presenting the results of this exact diagonalization, we discuss a certain decoupling approximation that has the advantage of showing explicitly how the decay of the quasiparticle is different from the diffusion on a Cayley tree.

The problem of the diffusion on a Cayley tree can be solved exactly because the self-energy satisfies a closed recursion relation $[10,11]$. Such a recursion relation exists because the Cayley tree has no loops. The lattice in Fock space generated by the quasiparticle decay process [3] does have loops, but we believe that these do not play an essential role and we will ignore them. The decoupling approximation consists in writing the self-energy $\Sigma_{i k l}(E)$ of a three-particle excitation as the sum of single-particle self-energies:

$$
\begin{aligned}
\Sigma_{i k l}(E)= & \Sigma_{i}\left(E-\bar{\varepsilon}_{k}-\bar{\epsilon}_{l}\right)+\Sigma_{k}\left(E-\bar{\varepsilon}_{l}-\bar{\varepsilon}_{i}\right) \\
& +\Sigma_{l}\left(E-\bar{\varepsilon}_{i}-\bar{\varepsilon}_{k}\right) .
\end{aligned}
$$

Here $\bar{\varepsilon}_{i}$ is the excitation energy, defined as $\bar{\varepsilon}_{i}=\varepsilon_{i}$ for an electron $(i \geq 0)$ and $\bar{\varepsilon}_{i}=-\varepsilon_{i}$ for a hole $(i<0)$. With this approximation, the self-energy satisfies the recursion relation

$$
\Sigma_{j}(E)=\sum_{k l} V_{i j, k l}^{2}\left[E-\bar{\varepsilon}_{i}-\bar{\varepsilon}_{k}-\bar{\varepsilon}_{l}-\Sigma_{i}\left(E-\bar{\varepsilon}_{k}-\bar{\varepsilon}_{l}\right)-\Sigma_{k}\left(E-\bar{\varepsilon}_{l}-\bar{\varepsilon}_{i}\right)-\Sigma_{l}\left(E-\bar{\varepsilon}_{i}-\bar{\varepsilon}_{k}\right)\right]^{-1},
$$

where the sum runs over the indices $k, l$ with $0 \leq k<l$ and $i=k+l-j<0$.

This recursion relation in Fock space can be compared with the recursion relation for the Cayley tree [10], which has the form

$$
\Sigma_{j}(E)=\sum_{k} V_{j, k}^{2}\left[E-\varepsilon_{k}-\Sigma_{k}(E)\right]^{-1} .
$$

Here the sum runs over all sites $k$ (energy $\varepsilon_{k}$ ) of the next level of the tree that are connected to $j$, with hopping matrix elements $V_{j, k}$. We notice two differences between Eqs. (7) and (8). The first is that the recursion relation on the Cayley tree conserves energy, while the recursion relation in Fock space does not. Another way of saying this is that Eq. (8) is a recursion relation between numbers $\Sigma_{j}$ at one fixed $E$, while Eq. (7) is a relation between functions $\Sigma_{j}(E)$. The second difference is that the number of self-energies coupled by repeated applications of the recursion relation in the Cayley tree grows exponentially (limited only by the size of the lattice), while in Fock space this number remains fixed at the number $2 j$ of singleparticle levels coupled to the excitation $c_{j}^{\dagger}|\mathrm{FS}\rangle$ by the interaction. Since $2 j$ is exponentially smaller than the size $\mathcal{P}(j)$ of the lattice in Fock space, this is an enormous difference with the Cayley tree. We are able to make such a precise statement because of the simplifications inherent to the layer model. However, we believe that the strong correlations between excitations with the same number of quasiparticles implied by Eq. (7) are present as well in the full problem of quasiparticle decay - although we cannot write down such a simple recursion relation for the full problem.

We have calculated the average single-particle density of states $\bar{\rho}_{j}(E)$ and the inverse participation ratio $P_{j}$ by exact diagonalization for $j$ up to 30 , as a function of the dimensionless conductance $g=\Delta / V$. [We have also computed the same quantities by numerically solving the recursion relation (7), and find good agreement.] The results for the average density of states collapse approximately onto the same curve (see Fig. 1), once the energies 


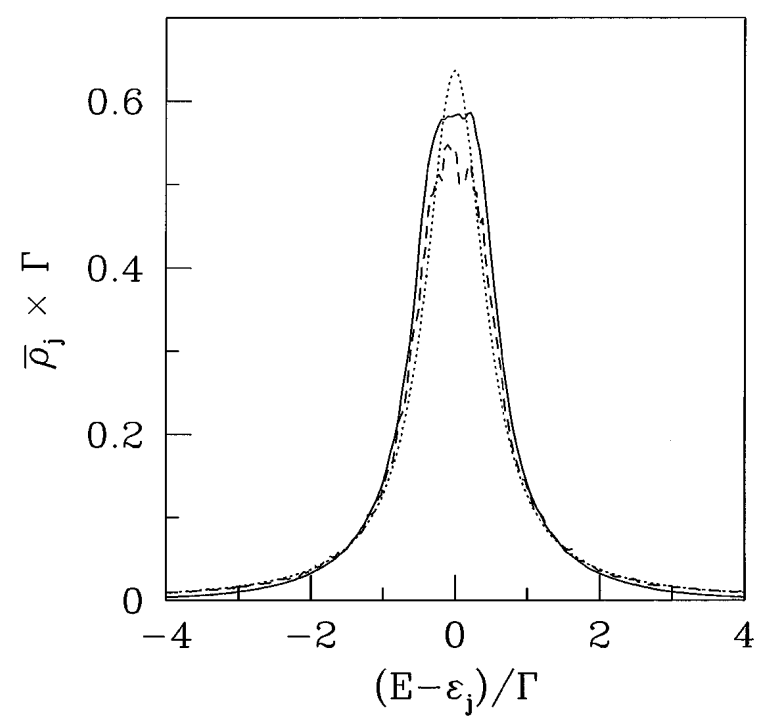

FIG. 1. Average single-particle density of states $\bar{\rho}_{j}(E)$, rescaled by $\Gamma=\frac{1}{3} \pi \Delta(j / g)^{2}$, for $j=25$. The solid and dashed curves are computed by exact diagonalization of the layer model for $g=55$ and $g=300$, respectively. Averages are taken over 7500 realizations of the random Hamiltonian. The dotted curve is a Lorentzian of unit area and width.

are rescaled by $\Gamma=\frac{1}{3} \pi \Delta(j / g)^{2}$. This expression for $\Gamma$ is the decay rate following from the golden rule of perturbation theory [2], assuming an energy-independent threeparticle density of states (equal to $\frac{1}{6} j^{2} / \Delta$ in the layer model). The small deviations from a Lorentzian (dotted curve in Fig. 1) are an artifact of the layer model. (They disappear if the restriction $i+j=k+l$ on the matrix elements $V_{i j, k l}$ of the interaction is removed.)

As expected, there is no indication in the average density of states $\bar{\rho}_{j}$ of a localization transition. The density of states $\rho_{j}$ for a single realization of $H$ is shown in Fig. 2. The difference between $\rho_{j}$ for small and large values of the ratio $\Gamma / V$ is striking: a small number of sharp, isolated peaks at large $\Gamma / V$ (top panel), in contrast to a single broad peak at small $\Gamma / V$ (bottom panel). Following the argument of Ref. [3], the top panel demonstrates that the single-particle excitation does not spread uniformly over the lattice in Fock space but remains localized at a small number of sites. The bottom panel is characteristic for an extended state in Fock space. Upon increasing $\Gamma / V$, there is therefore a transition from extended to localized states.

To study the localization transition we calculate the inverse participation ratio $P_{j}$, defined in Eq. (5). Following Ref. [9], we compare with the prediction of a totally delocalized situation ("golden rule"). The golden rule prediction is $P_{j} \simeq \min (1, \delta / \Gamma)$, where $\delta$ is the mean energy separation of the eigenstates $|\alpha\rangle$ of $H$. In the layer model, $\delta \simeq \Delta / \mathcal{P}(j)$. Since $\delta / \Gamma \propto g^{2}$, the golden rule predicts a quadratic increase of $P_{j}$ with increasing $g$, until $P_{j}$ saturates at a value of order unity [12]. A faster than quadratic increase is a signature of localization, in the sense that the
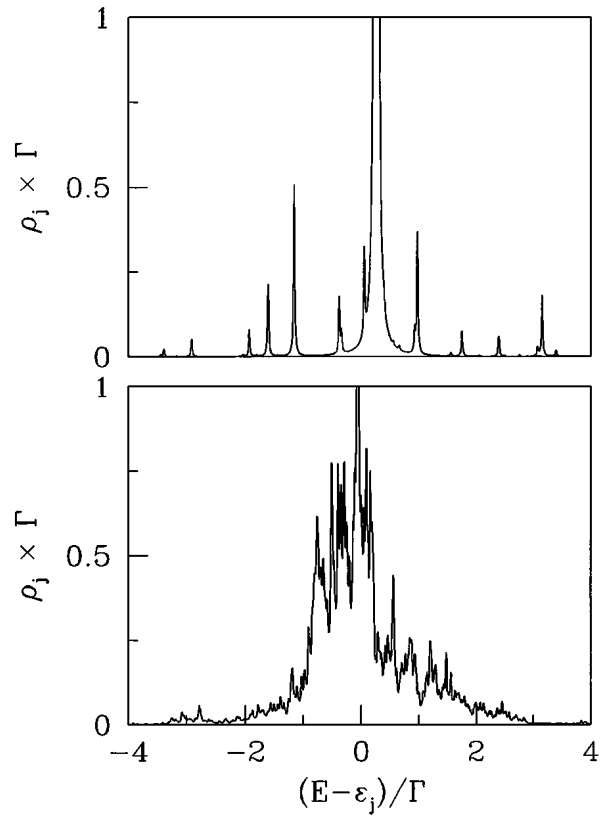

FIG. 2. Single-particle density of states $\rho_{j}$ of an individual member of the ensemble of quantum dots, computed by exact diagonalization for $j=25$ and two values of $g: 300$ (upper panel) and 55 (lower panel). The two results are qualitatively different, although the ensemble averages are essentially the same (see Fig. 1).

eigenfunctions do not extend over the entire lattice in Fock space. We show in Fig. 3 a double-logarithmic plot of $P_{j}$ versus $g$ for $j=15,20,25$, and 30. The straight lines of slope 2 show the quadratic increase predicted by the golden rule. The largest systems considered ( $j=25$, squares and $j=30$, crosses) have unambiguously a region of faster

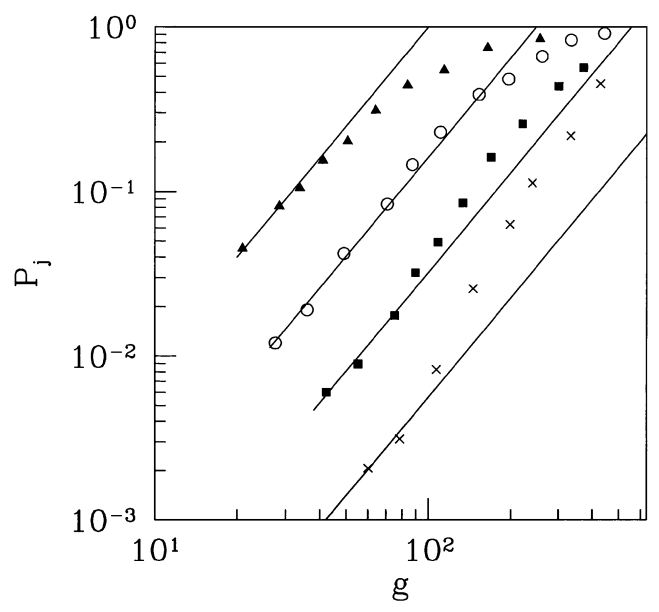

FIG. 3. Inverse participation ratio as a function of dimensionless conductance, for $j=15$ (triangles), $j=20$ (circles), $j=25$ (squares), and $j=30$ (crosses). The straight lines of slope 2 on the log-log scale show the quadratic increase of $P_{j}$ with $g$ predicted by the golden rule. A faster than quadratic increase indicates a transition to the localized regime. Statistical error bars have the size of the markers. 
than quadratic increase of $P_{j}$. In contrast, the smallest system considered ( $j=15$, triangles) follows the golden rule prediction until it saturates at $g \approx 60$. This system is clearly too small to show the transition to a localized regime. The largest system studied in Ref. [9] had $j \approx 15$, and indeed no deviations from the golden rule were found in that paper. We find that the inverse participation ratio exceeds the golden rule prediction by as much as a factor of 3 in our largest system. This excess builds up gradually with increasing $g$, consistent with the prediction $[5,6]$ of a smooth rather than an abrupt transition from extended to localized states.

In conclusion, we have studied a model for quasiparticle decay in a quantum dot that preserves the strong correlations omitted in the Cayley-tree model, yet remains tractable enough that large excitation energies are accessible. Our largest system demonstrates a breakdown of the golden rule of perturbation theory that had remained elusive in previous studies on smaller systems.

We thank P. W. Brouwer, J.-L. Pichard, and X. Waintal for helpful discussions. This work was supported by the European Community (Program for the Training and Mobility of Researchers) and by the Dutch Science Foundation NWO/FOM.

[1] U. Sivan, F. P. Milliken, K. Milkove, S. Rishton, Y. Lee, J. M. Hong, V. Boegli, D. Kern, and M. de Franza,
Europhys. Lett. 25, 605 (1994).

[2] U. Sivan, Y. Imry, and A. G. Aronov, Europhys. Lett. 28, 115 (1994).

[3] B. L. Altshuler, Y. Gefen, A. Kamenev, and L. S. Levitov, Phys. Rev. Lett. 78, 2803 (1997).

[4] D. Weinmann, J.-L. Pichard, and Y. Imry, J. Phys. I (France) 7, 1559 (1997).

[5] A. D. Mirlin and Y. V. Fyodorov, Phys. Rev. B 56, 13393 (1997).

[6] P. Jacquod and D. Shepelyansky, Phys. Rev. Lett. 79, 1837 (1997).

[7] P. G. Silvestrov, Phys. Rev. Lett. 79, 3994 (1997).

[8] R. Berkovits and Y. Avishai, Phys. Rev. Lett. 80, 568 (1998).

[9] C. Mejía-Monasterio, J. Richert, T. Rupp, and H. A. Weidenmüller, Phys. Rev. Lett. 81, 5189 (1998).

[10] R. Abou-Chacra, P. W. Anderson, and D. J. Thouless, J. Phys. C 6, 1734 (1973).

[11] A. D. Mirlin and Y. V. Fyodorov, Nucl. Phys. B366, 507 (1991).

[12] B. Georgeot and D. L. Shepelyansky, Phys. Rev. Lett. 79, 4365 (1997).

[13] Ya. M. Blanter, Phys. Rev. B 54, 12807 (1996).

[14] P. W. Brouwer (private communication).

[15] J. B. French and S.S.M. Wong, Phys. Lett. B 33, 449 (1970); 35, 5 (1971).

[16] O. Bohigas and J. Flores, Phys. Lett. B 34, 261 (1971); 35, 383 (1971).

[17] V. V. Flambaum and F. M. Izrailev, Phys. Rev. E 56, 5144 (1997). 